\title{
New Synthesis: Volatiles Bring out the Animal in Plants
}

\section{Erik H. Poelman}

Published online: 4 August 2013

(C) Springer Science+Business Media New York 2013

\author{
Keywords Plant-plant communication $\cdot$ Kin selection $\cdot$ Herbivore \\ induced plant volatiles
}

Until a decade ago, animal-based theory of communication appeared to have little common ground with that of plant communication. Because animals can discriminate relatives from unrelated individuals, they may use communication in a variety of social behaviors that result in increased inclusive fitness. Kin selection via altruistic behavior has long been considered to be beyond the capabilities of plants.

However, after work by Dudley and File in 2007, several studies identified that plants, through different kinds of root exudates, may recognize the presence of non-self root-systems and may even be able to discriminate root systems of kin and non-kin. When growing alongside competitors, focal and competing plants increase their root growth, but when growing next to kin, both the focal and neighboring plant do not. Although this does qualify as kin-selection (File et al. 2012), it differs from many animal altruistic communication forms in which a sender creates a message that informs a related receiver. Both plants in a communicating pair simultaneously function as sender and receiver, as is reflected in their growth responses when encountering a neighbor, and their messages often are continuously sent in the form of root exudates that mediate kin-recognition.

Studies on kin recognition in plants have remained belowground and have been focused on root exudates as a primary communication channel. Recently, Karban et al. (2013) provided support for kin recognition in plants mediated by airborne volatile organic compounds (VOCs). These semiochemicals play key roles in the interactions between plants and herbivorous attackers, beneficial pollinators and predators, and serve a dual function as a fast-track communication system within the plant when organs are more distantly connected through the vascular system. The same VOCs may be 'eavesdropped' upon by conspecific neighboring receiver plants, which may benefit from their information content when it predicts future attack, for example, by herbivorous insects. Receiver plants exposed to herbivore-induced VOCs can be thought to "anticipate" herbivore attack by priming or enhancing their state of defense. Karban et al. (2013) clipped sage brush plants to mimic herbivory and found that receiver plants that were closely related to the emitter responded more strongly to the information and became more resistant to herbivory than non-kin neighbors. In this study, clearly the emitter showed plasticity in its provision of information, and the information resulted in stronger beneficial responses of kin receivers than non-kin receivers, which satisfies many of the criteria for altruistic communication as defined for kin selection in animals.

E. H. Poelman $(\bowtie)$

Laboratory of Entomology, Wageningen University, PO Box 8031, 6700 EH Wageningen, The Netherlands

e-mail: erik.poelman@wur.nl
Nevertheless, animal kin selection often includes the condition that the sender is aware of its neighborhood and only provides information when the neighbor is identified as kin. In Karban's study, the emitters were not tested for context dependency of their information provisioning. However, from studies of both above and belowground plant communication there is support that plants may adapt their provision of information conditional upon their neighborhood. Muroi et al. (2011) found that lima bean plants that had detected the herbivore induced VOC $(E)-\beta$-ocimene from an emitter plant, responded more strongly to spider mite infestation than lima bean plants that had not received this message. Upon infestation by spider mites, the ocimene-receiver plants were not only more resistant to spider mites, but also their spider mite-induced volatiles in turn induced resistance more effectively on a second lima bean receiver than spider mite induced volatiles of receivers that had not been exposed to $(E)-\beta$ ocimene. Hence, lima bean plants that perceived the context of herbivory, communicated the risk of herbivory more strongly to their own neighbors. The presence of both awareness of neighborhood and the ability to differentially communicate with kin and non-kin may endow plants with an animal-like repertoire of responses that can promote kin selection.

To set the boundaries of where plant and animal altruistic behaviors differ, future studies should stretch the limits of the animal side in plants and explore the potential that both emitters and receivers have in plant-plant communication. One important aspect is whether emitters are capable of responding differently to the same stressor in a community of kin vs. a community of non-kin. In other words, are plants capable of keeping quiet when standing in the wrong crowd? Just as vervet monkeys use distinct vocalizations as alarm calls distinguishing avian predators from snakes, plants respond to attack from different kinds of herbivores by emitting specific VOC blends. The specificity of herbivore-induced plant VOC release may allow receivers to anticipate the feeding guild or species of herbivore that is likely to attack them. We have yet to explore what level of detail plants can extract from the message released by the emitter, and whether these detailed messages can specifically benefit kin receivers.

\section{References}

File AL, Murphy GP, Dudley SA (2012) Fitness consequences of plants growing with siblings: reconciling kin selection, niche partitioning and competitive ability. Proc R Soc B 279:209-218

Karban R, Shiojiri K, Ishizaki S, Wetzel WC, Evans RY (2013) Kin recognition affects plant communication and defence. Proc R Soc B 280:20123062

Muroi A, Ramadan A, Nishihara M, Yamamoto M, Ozawa R, Takabayashi J, Arimura G-I (2011) The composite effect of transgenic plant volatiles for acquired immunity to herbivory caused by inter-plant communications. PLoS One 6:e24594 